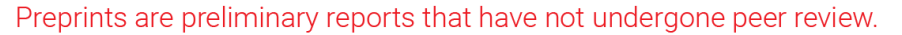 They should not be considered conclusive, used to inform clinical practice, or referenced by the media as validated information. \\ On the dual effect of obstacles in preventing silo clogging
}

\section{Diego Gella ( $\nabla$ dgella@unav.es )}

Universidad de Navarra https://orcid.org/0000-0001-5040-6728

\section{Daichi Yanagisawa}

The University of Tokyo

\section{Rodrigo Caitano}

Universidad de Navarra https://orcid.org/0000-0003-1481-1392

\section{Maria Victoria Ferreyra}

Universidad Nacional de La Pampa

Iker Zuriguel

Universidad de Navarra

\section{Article}

Keywords: fluid dynamics, clog formation, geometrical effects, kinematic effects

Posted Date: June 9th, 2021

DOl: https://doi.org/10.21203/rs.3.rs-580101/v1

License: (c) (1) This work is licensed under a Creative Commons Attribution 4.0 International License. Read Full License

Version of Record: A version of this preprint was published at Communications Physics on January 10th, 2022. See the published version at https://doi.org/10.1038/s42005-021-00756-4. 


\title{
On the dual effect of obstacles in preventing silo clogging.
}

\author{
Diego Gella ${ }^{1, *}$, Daichi Yanagisawa ${ }^{2,3}$, Rodrigo Caitano ${ }^{1}$, María Victoria Ferreyra $^{4}$, and \\ Iker Zuriguel $^{1}$
}

1Departamento de Física y Matemática Aplicada, Facultad de Ciencias, Universidad de Navarra, Pamplona, Spain
${ }^{2}$ Research Center for Advanced Science and Technology, The University of Tokyo, Tokyo, Japan
${ }^{3}$ Department of Aeronautics and Astronautics, School of Engineering, The University of Tokyo, Tokyo, Japan
${ }^{4}$ Departamento de Física, Facultad de Ciencias Exactas y Naturales, Universidad Nacional de La Pampa, Santa
Rosa, Argentina
*dgella@unav.es

\section{ABSTRACT}

We experimentally demonstrate that an obstacle, when suitably placed above a bottleneck, leads to a reduction of clog formation in a silo via two different mechanisms. The first one, already suggested in previous works, is related to an alteration of the kinematic properties in the outlet proximities that prevents the stabilization of arches. The second, that is discovered when working at a quasi-static regime, appears because the obstacle induces a clear anisotropy in the contact fabric tensor. Then, both mechanisms are encompassed using a single equation in which two parameters -one related with the geometrical effects and the other to the kinematic ones-are enough to reproduce all the experimental results.

\section{Introduction}

The placement of obstacles has been suggested as a smart strategy to prevent clog formation when a discrete system flows through a constriction. This a priori counter-intuitive behavior, was originally proposed to improve pedestrian evacuation from a room with a small exit ${ }^{1}$. After this work, the increment of the flow rate by suitably placing an obstacle in front of the gate has been taken as a hallmark of pedestrian dynamics and, accordingly, it has been reproduced by most of the recently proposed models $^{2-8}$. Nevertheless, a robust experimental demonstration of the obstacle effectiveness in pedestrian dynamics is still lacking given that the existing results are contradictory ${ }^{9-15}$. Paradoxically, this experimental evidence exists for other systems as i) the passage of animals such as sheep ${ }^{16,17}$ or mice ${ }^{18}$ through narrow doors, and ii) the discharge of granular matter from a silo ${ }^{19-22}$.

It is precisely the granular scenario the one that allows a better and more detailed analysis of the system behavior ${ }^{23-26}$; first because of the simpler interaction rules among inert grains, and second because it allows the performance of as many as experimental repetitions as desired. In this way, it has been found that, placed at the optimal distance from the orifice, a circular obstacle reduces the clogging probability more than one hundred times without affecting the flow rate ${ }^{19,20}$. When the obstacle position is raised above the optimal location the effect progressively reduces, whereas if it is placed too close to the outlet, clogging increases abruptly as arches start to form between the obstacle and the silo bottom instead of above the orifice ${ }^{19}$.

Furthermore, experiments of grains discharging from a silo have revealed that the key aspect behind clogging reduction is the creation of an empty region just below the obstacle ${ }^{21}$. Thus, when a group of particles coincide above the orifice and form an arch, its stabilization becomes very difficult in comparison with the case without obstacle (where the beads above the arch restrict the motion of the particles conforming $\mathrm{it}^{19}$ ). An indirect quantification of this behavior consisted in computing the ratio of particles moving upwards in the outlet region with respect to the total, a magnitude that revealed a net correlation to clogging reduction: the higher the ratio of upwards displacements, the lower the probability of clogging. In addition, Endo et al. ${ }^{21}$ reported an associated effect of the obstacle on clogging reduction consisting in increasing the granular temperature of the sample in the horizontal direction.

In conclusion, the existing explanations that justify the effect of an obstacle on clogging are related with the system dynamics. Therefore, it could be expected that if the grains motion is minimized, the obstacle impact will become barely important. In this work, we demonstrate that this is not the case by implementing a granular extraction mechanism consisting in a belt placed below the orifice that permits discharging the silo quasistatically ${ }^{27}$ (see Methods). Indeed, we discover that in quasi-static discharges, the presence of the obstacle introduces a clear anisotropy in the contact fabric tensor that justifies the reduction of clogging probability. 


\section{Results}

The main objective of this work is to investigate how the presence of the obstacle above the exit of a silo affects the emergence of clogging when the kinematic effects are progressively reduced (up to the quasi-static limit). For this purpose, we performed experiments in a two-dimensional silo like the one shown in Fig. 1a. There, both the obstacle above the orifice and the conveyor belt to extract the material are appreciated. An automated measurement protocol, visually explained in a movie included as Suppl. Material, enables the computation of the clogging probability $p_{c}$ from the avalanches distribution in each experimental configuration. Additional kinematic information is extracted from video recordings (see Methods), a single frame of which is shown as an example in Fig. 1 b.

Although changes in kinematics are achieved by means of tuning the velocity of the extracting belt $\left(v_{b}\right)$, the parameter that we will use here to characterize the system dynamics is $v$, the particles velocity at the very outlet (more specifically, in the blue box displayed in Fig. 1b). This strategy was proved to be useful in previous works ${ }^{27}$ permitting a successful encompassing of the results obtained for different gaps among the belt and the silo bottom (the other parameter that affects the particle velocities apart from the belt velocity ${ }^{28}$ ). Indeed, in agreement with the findings reported in these works for standard silos, when the obstacle is placed above the orifice we find a non-linear relationship among the particle velocities at the outlet and the belt velocity (Fig. 1c). Note that, as depicted in Fig. 1b, grains moving downwards have positive velocities. For low $v_{b}$ the dependence is rather linear because the belt is mainly controlling the extraction dynamics; but then, as $v_{b}$ increases, the orifice starts to screen the grains passage and their velocity saturates in what it was called the "free discharge regime" because of its similarity with a scenario in which the discharge is just driven by gravity (without belt). As expected, the saturation value is lower for the smaller outlet size $(D)$ as in a freely discharged silo the velocities of the grains at the outlet scale with $D^{1 / 2} 29$.
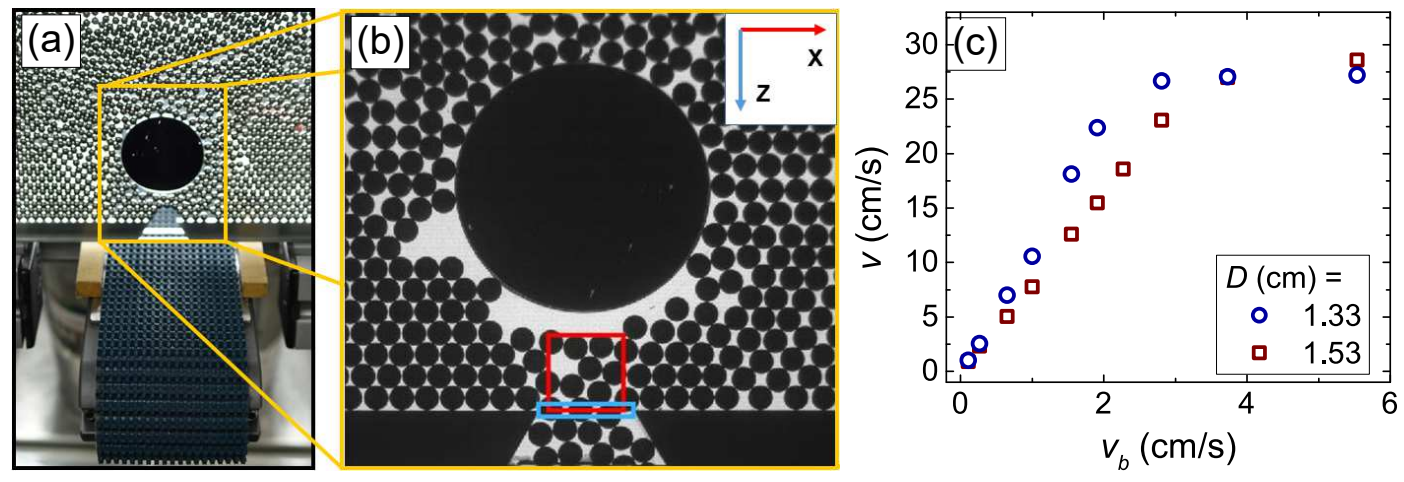

Figure 1. (a) Photograph of the bottom part of the silo where the obstacle, the orifice and the conveyor belt can be distinguished. (b) Frame taken from a film recorded with the high-speed camera. The blue box is the region located at the outlet where the velocity of particles is averaged to compute $v$. The red box is the representative area above the orifice and below the obstacle where the magnitudes are averaged to obtain $v_{x}, v_{z}$ and $\phi$. The coordinates system (where for convenience, positive values are considered in the downwards direction) is also shown in this figure. (c) Experimental data of the mean velocity of the particles when exiting the silo $v$, versus the belt velocity $v_{b}$, in the silo with the obstacle, for the two orifice sizes indicated in the legend.

\section{Clogging}

Once we know the characteristic velocity of the particles when crossing the exit for each experimental condition, we compute the corresponding clogging probability $p_{c}$. This magnitude, which accounts for the probability that a particle clogs the system when it is exiting the silo, can be easily calculated from the exponential distribution of avalanche sizes (measured as the number of particles, $s$, flowing out of the silo before an arch produces a clog $)^{24-27}$. Clearly, Fig. 2 a reveals a decreasing trend of $p_{c}$ with $v$, a result that qualitatively resembles the one obtained without obstacle ${ }^{27}$. Indeed, for the two outlet sizes investigated, the curves can be successfully fitted with the expression proposed in that work:

$$
p_{c}=(a+b v)^{-\left(D / d_{p}\right)^{2}}
$$

in which, $d_{p}$ is the particle diameter, $v$ the velocity of the grains, and $b$ and $a$ are two fitting parameters that account, respectively, for the effect of the system dynamics and geometrical properties of arches.

Remarkably, Fig. 2a evidences a systematic deviation between the data obtained with and without obstacle: the obstacle reduces the clogging probability to less than $50 \%$ of the reference value without obstacle for all velocities, including the limit 
scenario of a quasi-static discharge (i.e. $v \rightarrow 0$ ). This reflects on the different fitting parameters obtained when the obstacle is present $\left(a=1.41\right.$ and $\left.b=0.015 \mathrm{~s} \mathrm{~cm}^{-1}\right)$ than when it is not $\left(a=1.33\right.$ and $\left.b=0.0128 \mathrm{~s} \mathrm{~cm}^{-1}\right)$. Interestingly, both, the geometrical and dynamics parameters vary when placing the obstacle, suggesting a dual role of it in what concerns clogging prevention. Indeed, although the explanations given in the literature about the obstacle role were related to an alteration of the system dynamic properties near the orifice, it is rather clear that, at the quasi-static limit, there is a net difference among the two pairs of curves shown in Fig. 2a.

Aiming to confirm this feature and to evaluate its generality when the outlet size is varied, we implemented a series of experiments at the quasi-static limit (Fig. 2b). Univocally, the presence of the obstacle leads to a reduction of the clogging probability even when the particle dynamics is expected to play a negligible role. Consistently, the results obtained can be nicely fitted with Eq. 1 using $v=0$ and then, a single fitting parameter $a=1.41$. Of course, the value of $a$ is the same than the one obtained when fitting the $p_{c}$ vs $v$ curves for the scenario with obstacle in Fig. $2 \mathrm{a}$.

From these results, we can infer that the obstacle should have a dual effect on the system properties: on one side, it may affect the geometrical arrangements occurring in the quasi-static regime, but on the other, it should also lead to an alteration of the system dynamics. Therefore, in order to confirm this idea, we will further analyse the effect of the obstacle in several kinematic and static (geometrical) magnitudes that may be related with the development of clogging.
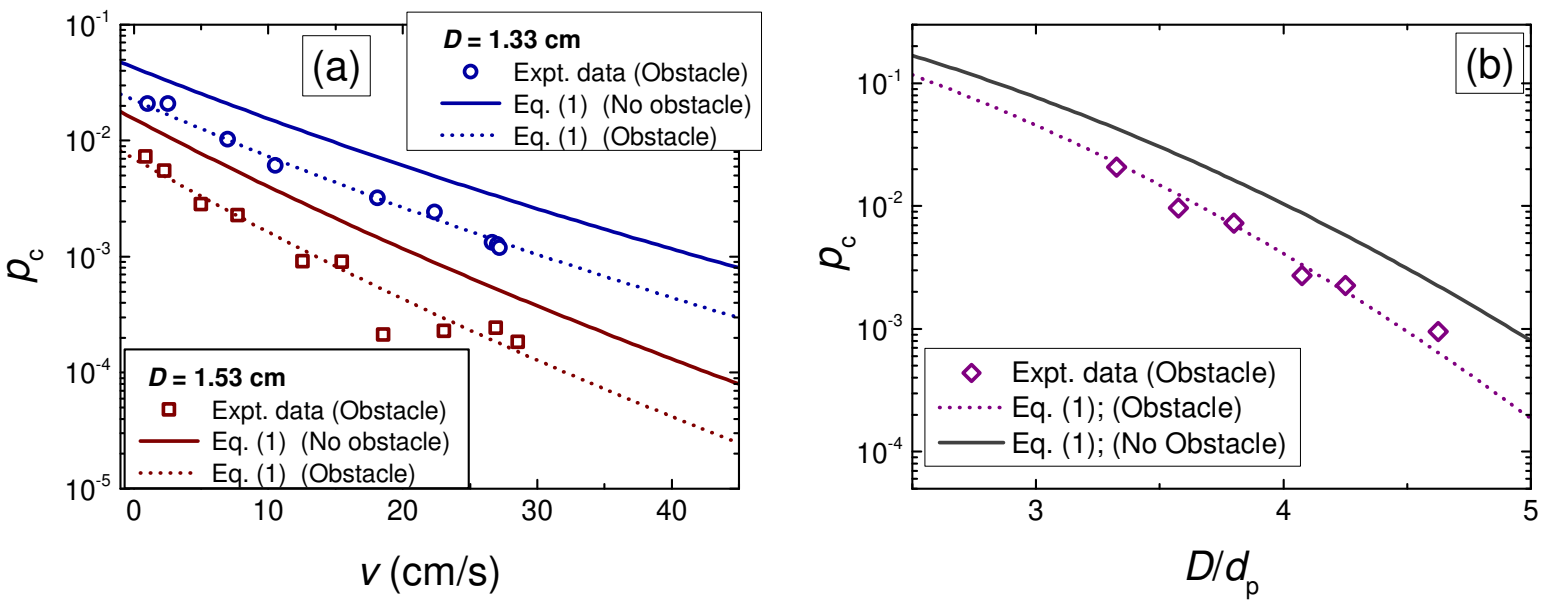

Figure 2. Probability of clogging as function of the mean velocity of particles $v$ at the orifice, for the orifice sizes shown in the two legends. The solid lines are representations of Eq. 1 with $a=1.33$ and $b=0.01280 \mathrm{sm}^{-1}$, the parameters found in Ref. $^{27}$ for a silo without obstacle. Dotted lines correspond to the same expression with $a=1.41$ and $b=0.015 \mathrm{~s} \mathrm{~cm}{ }^{-1}$, the parameters corresponding for the obstacle position implemented in this work. (b) Probability of clogging as function of the aspect ratio $D / d_{p}$ in the quasi-static regime. Purple diamonds correspond to experimental data while solid and dashed lines are representations of Eq. 1 with the same values of $a$ introduced above for the obstacle and no-obstacle cases. Note that the value of $b$ in Eq. 1 is irrelevant in the quasistatic regime as $v \rightarrow 0$.

\section{Dynamics}

In this section, we will explore the behavior of magnitudes related to the system dynamics in the region between the obstacle and the orifice; i.e. the position at which clogging arches develop. We start displaying in Figs. 3a,b the distributions of particles velocities, both in the horizontal direction $\left(v_{x}\right)$ and in the vertical one $\left(v_{z}\right)$. Clearly, the distributions of $v_{x}$ are slightly wider for the scenario with the obstacle than without it, a result that can be understood if we think that the obstacle forces the particles to detour around it, hence increasing the horizontal component of the velocity and reducing the vertical one. This can be effectively corroborated by looking at the distributions of vertical velocities, which evidence that the obstacle leads to a small reduction of large positive velocities (recall that downwards velocities have positive values). Interestingly, this feature is accompanied by a remarkable widening of the distribution tails towards negative values. Indeed, for the cases without obstacle all these tails seem to fall relatively on top of each other, whereas when the obstacle is placed, they seem to broaden as the extraction rate augments. From this, we can conclude that the presence of the obstacle favours the upwards motion of the particles above the orifice. This feature, which was already related to an arch destabilization process in a freely discharged silo ${ }^{27}$, seems to be more important as the extraction rate and grains velocities increase.

Then, in order to confirm this instance and better characterize the effect of the obstacle in the particle dynamics when the velocity of the grains crossing the exit $v$ is varied, $\left\langle\left|v_{x}\right|\right\rangle$ and $\left\langle v_{z}\right\rangle$ are represented (in Figs. 3c,d respectively) as a function 
of $v$. The detouring of particles around the obstacle explained above is confirmed as $\left\langle\left|v_{x}\right|\right\rangle$ augments and $\left\langle v_{z}\right\rangle$ reduces in the obstacle presence. This feature becomes more important when $v$ increases as the difference among the obstacle and no-obstacle curves is enhanced. Complementarily, the upwards motion of the grains in the outlet vicinity is characterized by computing the number of upwards displacements $V_{Z}^{-}$with respect to the total number of displacements detected $V_{Z}^{T}$ in the analysed region; or, in other words $\int_{-\infty}^{0} p d f\left(v_{z}\right) d v_{z} / \int_{-\infty}^{\infty} p d f\left(v_{z}\right) d v_{z}$. The dependence of this proportion is represented as a function of the grains exit velocity in Fig. 3e revealing an interesting behavior. In absence of the obstacle, the proportion of upwards displacements for high exit velocities is negligible but, when reducing $v$, this augments considerably up to almost $15 \%$ for the lowest value of $v$. The same curve shape is observed for the scenario with the obstacle, but in this case all data are shifted upwards; i.e. the obstacle favours upwards displacements when $v$ is high. In addition, when $v$ is reduced the growth of $V_{Z}^{-} / V_{Z}^{T}$ is less pronounced for the scenario with the obstacle, hence provoking that both curves approach each other when $v \rightarrow 0$. From this, we can interpret that the effect of the obstacle in the kinematic properties of the system above the orifice is minimized when reducing the velocity with which the particles are extracted from the silo. If this is the case, the reason for which the obstacle considerably reduces the clogging probability in the quasi-static limit as observed in Fig. 2, remains undisclosed.

A first indicator that would justify a possible origin of the clogging reduction in the quasi-static limit comes from the analysis of the two-dimensional solid fraction $\phi$ in the region comprised between the exit and the obstacle. Indeed, as shown in Fig. 3f, the obstacle presence induces the formation of an empty gap below it, reducing the solid fraction in around 0.1 units. Interestingly, this effect seems to be rather independent on the extraction velocity, hence persisting for the quasi-static limit. Therefore, lower packing fractions would correspond to reduced probabilities of particles touching each other above the outlet, and then to a smaller probability of getting stable arches formed. Alternatively, lower packing fractions can be also assumed to give rise to smaller pressures at the arch formation region, hence preventing the arch stabilization.
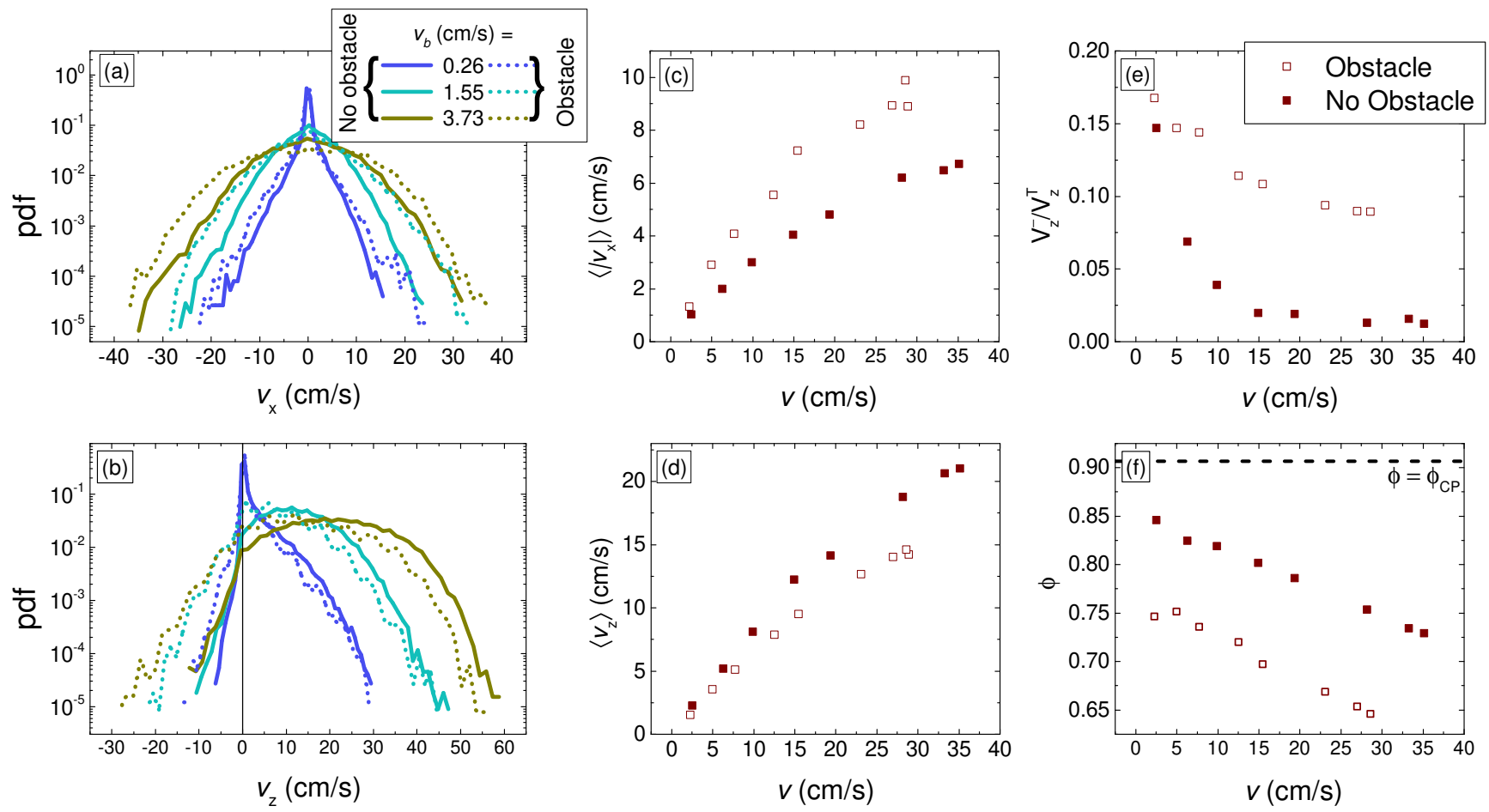

Figure 3. Experimental probability density functions (pdf) of (a) horizontal and (b) vertical velocity components measured in the region indicated with a red box in Fig. $1 \mathrm{~b}$ for an orifice size of $D=1.53 \mathrm{~cm}$ and the belt velocities indicated in the legend. Dashed lines account for the experiments with the obstacle while solid ones make reference to the experiments without it. In (c) and (d) the averages of these distributions (using the absolute value in the case of $v_{x}$ ) are reported as a function of $v$, the particles velocities when crossing the outlet. (e) Proportion of upwards displacements (computed as the number of negative velocities $V_{Z}^{-}$divided by the total number of velocities $V_{Z}^{T}$ analyzed in the red box of Fig. 1b) versus the particles velocities when crossing the outlet. (f) Two-dimensional solid fraction in the area signaled with a red box in Fig. $1 \mathrm{~b}$ as a function of the particles velocities when crossing the outlet. The dashed line shows $\phi_{C P}=\pi \sqrt{3} / 6$, the maximum value available for $\phi$ in this system which corresponds to the close packing of circles in 2D. 


\section{Statics}

From the findings reported above, a better characterization of the quasi-static regime seems necessary. Therefore, in this section we will spatially analyse the particle-particle contacts in the outlet region for the obstacle and no-obstacle scenarios. To this end, we have evaluated the whole registered region for several static configurations. As the flow is intrinsically intermittent ${ }^{30}$, in order to avoid sampling repeatedly identical arrangements, we have decided to explore the system every time the flow is temporarily arrested (without a clogging arch formed) after a rearrangement. In Fig. 4a we show the temporal evolution of the kinetic energy $E_{k}$ in the system, defined as the sum of the kinetic energy of all beads in the visual field of the camera in each frame. The specific moments we have considered for the analysis are indicated with circles; as illustrated, one configuration is evaluated every time that the grains rearrange and stop again.
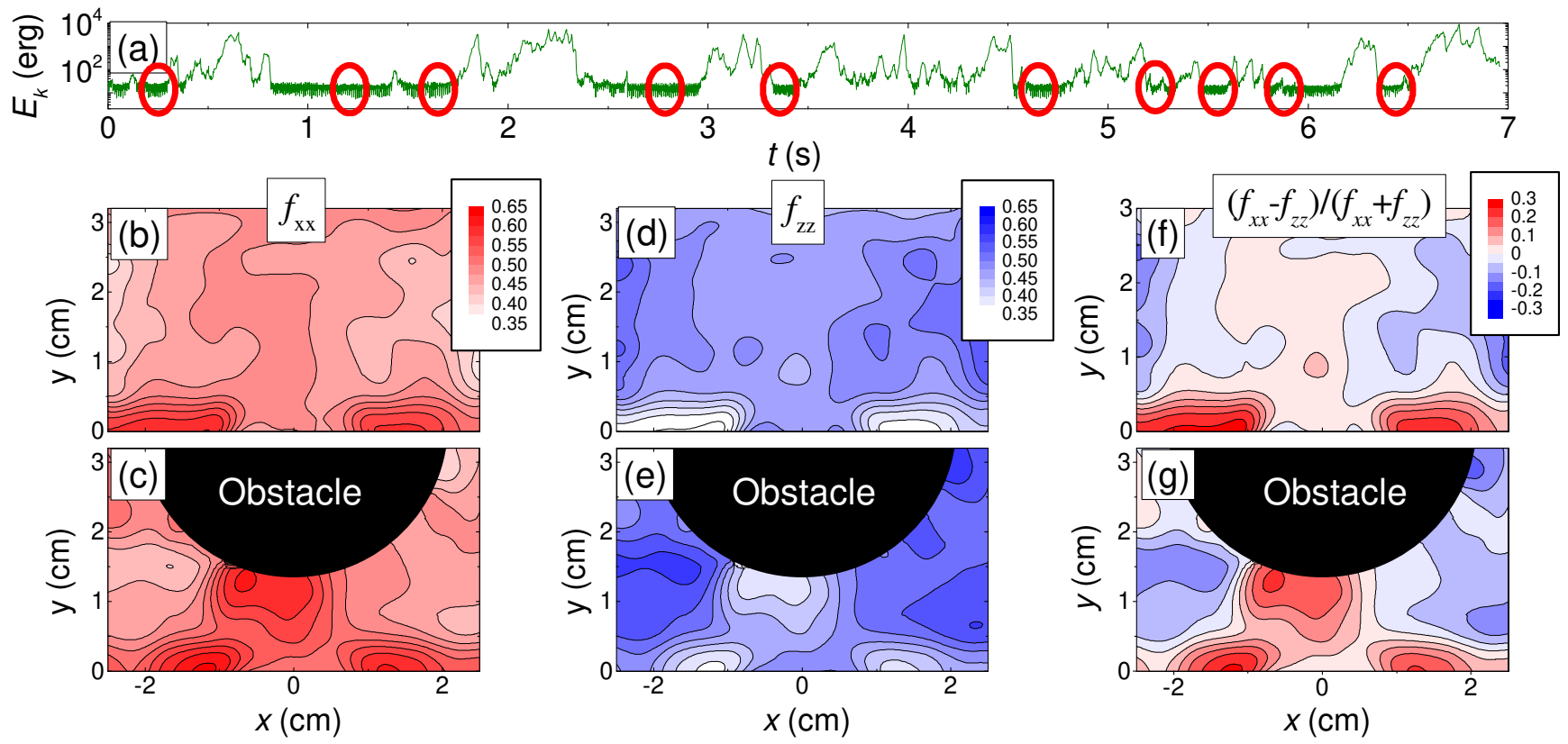

Figure 4. a) Example of the temporal evolution of the kinetic energy of the grains in the whole recorded region (above the orifice) for the quasi-static regime. Red circles indicate the moments at which the fabric tensor is analyzed. (b-e) Maps of the horizontal $f_{x x}$ and vertical $f_{z z}$ components of the fabric tensor averaged over around 40 different configurations. The results correspond to a silo with an orifice size of $D=1.53 \mathrm{~cm}$ without obstacle (top row panels) and wit it (bottom row panels). (f-g) Corresponding maps of the rescaled difference between the horizontal and vertical components of the contact fabric tensor $\left(f_{x x}-f_{z z}\right) /\left(f_{x x}+f_{z z}\right)$.

To assess the network orientation of contacts we computed the second order fabric tensor, which is defined for each particle $i$ as $f_{\alpha \beta}^{i}=\frac{1}{N_{c}} \sum_{c} n_{\alpha}^{c} n_{\beta}^{c}$. Here, $N_{c}$ is the number of contacts of that particle, while $\overrightarrow{n^{c}}$ is the normalized branch vector that links the centre of the particle $i$ with the ones of its contacting particles. From these data, we built continuous fields of this magnitude (see Methods) which result to be strongly dependent on the obstacle presence. The maps of the horizontal component of the fabric tensor $\left(f_{x x}\right)$ reveal a notable increase of this magnitude in the region below the obstacle (Fig. 4c), a behavior that is clearly coupled with a reduction in the values of the vertical component $f_{z z}$ (Fig. $\left.4 \mathrm{e}\right)$. Then, the roughly similar values of $f_{x x}$ and $f_{z z}$ developed above the orifice for the no-obstacle scenario that correspond to isotropic arrangements, become unbalanced due to the presence of the obstacle. This suggests that the obstacle introduces a marked anisotropic distribution of contacts, a behavior that is better reflected by representing the rescaled difference between the horizontal and vertical components of the contact fabric tensor $\left(\left(f_{x x}-f_{z z}\right) /\left(f_{x x}+f_{z z}\right)\right)$. Effectively, the values obtained above the outlet are close to zero in the no-obstacle configuration (Fig. 4f), whereas patently take positive values due to the obstacle weight screening (Fig. $4 \mathrm{~g}$ ).

\section{Discussion}

In summary, in this work we have experimentally demonstrated that the presence of an obstacle above a silo exit prevents clog formation due to the influence in both, the static arrangements and the particle dynamics. The former would be determinant in 
quasi-static discharges, whereas the latter would prevail for high extraction rates or free discharges (without belt). Consequently, our results evidence two physical mechanisms behind clogging reduction by the presence of an obstacle. The first one involves an alteration of the contact fabric tensor which, in the presence of the obstacle, becomes strongly anisotropic above the orifice. This anisotropy would reduce the probability of arch formation reached in a scenario without obstacle as an isotropically charged arch is more stable than an arch charged mostly from the sides. The second mechanism relates with the empty gap formed below the obstacle and the augment of horizontal velocities in this region. The combination of these two dynamical features provokes that when particles coincide above the orifice, their stabilization forming a clogging arch is very difficult: the horizontal collision and lack of confinement in the vertical direction impedes a complete dissipation of the energy and particles are ejected upwards.

Interestingly, all this complex behavior can be encompassed with a single equation (proposed before for the silo without obstacle) that describes the clogging probability as a function of particles velocities and the outlet size, using only two fitting parameters. As these two parameters (one related with the particles dynamics and the other with the quasi-static condition) are different for the obstacle and no-obstacle case, we speculate a dependence of them on the obstacle properties and position that should be studied elsewhere.

Finally, let us point out that the findings discovered in this work may help to shed light on the sometimes contradictory role that an obstacle has in other systems such as pedestrians, animals or active matter in general. Importantly, from a physical point of view, the analogy with a freely discharged silo does not seem the best choice for these systems, which are characterized by having a limit velocity instead of moving under a constant acceleration field; hence resembling the case of a silo discharged with a conveyor belt more than the freely discharged one.

\section{Methods}

\section{Experimental setup}

The system is a two-dimensional silo built by sandwiching two parallel glass sheets of $70 \mathrm{~cm}$ wide and $160 \mathrm{~cm}$ high. Between them, two $0.4 \mathrm{~cm}$ thick aluminium gauges supplemented by thin cardboards play the role of lateral sides. Then, only a single layer of granular material, $d_{p}=0.4 \mathrm{~cm}$ diameter spheres of AISI 420 stainless steel (density $\rho_{M}=7.75 \mathrm{gcm}^{-3}$ ), fits inside the panes. The silo bottom consists of two wedge-shaped stainless steel pieces whose separation defines the outlet size $D$ through which the grains flow out. The conveyor belt is placed below the orifice, with a separation of $0.67 \pm 0.03 \mathrm{~cm}$ between their upper protrusions and the lowest part of the steel pieces of the silo bottom. This device can operate in a range of belt velocities between 0.1 and $16 \mathrm{~cm} / \mathrm{s}$. In some runs, a circular obstacle $(4 \mathrm{~cm}$ diameter) made of methacrylate has been glued at a vertical distance of $1.56 \pm 0.03 \mathrm{~cm}$ from its lowest edge to the orifice centre. In the x-direction, the obstacle has been centred with the orifice. Additionally, a hopper situated at the top permits the manual filling of the silo.

\section{Clogging probability}

The probability that a bead clogs the system when it is crossing the orifice, $p_{c}$, is computed from the distribution of avalanche sizes $s$, defined as the amount of material discharged between two clogging events. In particular, the values of $p_{c}$ correspond to the negative slopes of the survival functions (ccdf) of $s$ in semi-logarithmic scale, taking into account that $\ln [\operatorname{ccdf}(s)] \simeq-s p_{c}$ (see Ref. ${ }^{33}$ for a detailed explanation). To obtain these distributions, in each experimental configuration, an automated measurement protocol synchronizes several devices which have been incorporated to the system: a balance with a bucket on it placed at the end of the belt, a web camera, and a vibration system to break the clogging arches. Once the silo is manually filled, the protocol is launched. Its steps are summarized in a video included as Suppl. Material and detailed hereafter. First, the balance is tared and the conveyor belt starts running at the desired velocity, discharging the granular material. During this time, the web camera is continuously evaluating the sum of pixels in a region located just below the area in which arches form. Then, if a clogging event occurs, there will be no beads at that area and the pixels will be mostly white, leading the sum above a predetermined threshold. If this situation carries on during four seconds, the arch is considered as stabilized. When this occurs, the belt pours all the grains discharged from the silo into the bucket and stops. Then, the avalanche size is registered by the balance, the shaker vibrates to break the arch, and the conveyor belt resumes its motion. This process is repeated until having around 1000 avalanches per experimental configuration. In all cases, the survival function of the avalanche sizes is computed and the corresponding value of $p_{c}$ is extracted from a linear fitting of the natural logarithm of these distributions.

\section{Kinematic and geometrical magnitudes}

Using a Photron ®FASTCAM-1024PCI high speed camera, we recorded several films of the grains motion at the area surrounding the orifice and the obstacle (see Fig. 1b). High-contrasting images are achieved thanks to a LED panel at the rear which provides backlighting illumination. For each experimental configuration, three videos have been recorded with a frame rate of $500 \mathrm{fps}$ and a duration of around 7 seconds. A home-made image processing software has been developed to detect the bead centres and track their trajectories. From their centres and radii, the particles are reconstructed in each frame, 
allowing the computation of the two-dimensional solid fraction as the proportion of space occupied inside a characteristic area. Furthermore, the particle velocities are calculated as the ratio between their displacements and the time between two frames $(1 / 500 \mathrm{~s})$. Finally, the contact fabric tensor has been computed from the branch vectors between contacting particles, defined as the ones whose centres are separated less than $0.41 \mathrm{~cm}$ (a little more than a particle diameter). Then, a coarse-grained method inspired in the work of Gorldhirsch ${ }^{34}$ has been used to build macroscopic continuous fields. Given the contact fabric tensor data linked to each position and time, $f_{\alpha \beta}^{i}(t)$, the mean field has been computed as $f_{\alpha \beta}(\vec{r}, t)=\frac{\sum_{i} f_{\alpha \beta}^{i}(t) \Theta\left(\vec{r}-\vec{r}_{i}(t)\right)}{\Theta\left(\vec{r}-\vec{r}_{i}(t)\right)}$. In this expression, $\Theta\left(\vec{r}-\vec{r}_{i}(t)\right)$ is the coarse-graining function, a $2 \mathrm{D}$ Gaussian function, $\Theta\left(\vec{r}-\vec{r}_{i}(t)\right)=\frac{1}{2 \pi w^{2}} \exp \left(-\left|\vec{r}-\vec{r}_{i}\right| / 2 w^{2}\right)$ with $w=d_{p} / 2$. For computational reasons, this function has been cut off for distances larger than $3 w$ from each particle centre. Finally, $f_{x x}$ and $f_{y y}$ have been averaged over all frames considered to obtain the amounts represented in the article, $f_{x x}(\vec{r})=\left\langle f_{x x}(\vec{r}, t)\right\rangle$ and $f_{y y}(\vec{r})=\left\langle f_{x x}(\vec{r}, t)\right\rangle$.

\section{Acknowledgements}

We thank D. Maza, A. Garcimartín, R.C. Hidalgo, and D. Hernández-Delfin for discussions and comments, as well as L.F. Urrea for technical support. We acknowledge support from Spanish Government through Project FIS2017-84631-P, MINECO/AEI/FEDER, UE. R.C. acknowledges Asociación de Amigos, Universidad de Navarra, for his grant.

\section{Author contributions statement}

I.Z. conceived the experiment(s), D.G., D.Y., R.C. and M.F. conducted the experiment(s) and analysed the results. I.Z. and D.G. wrote the manuscript that was thereafter reviewed by all authors.

\section{Competing interests}

The authors declare no competing interests.

\section{References}

1. Helbing, D., Farkas, I., and Vicsek, T., Simulating dynamic features of escape panic, Nature 407, 487-90 (2000).

2. Kirchner, A., Nishinari, K., and Schadschneider, A., Friction effects and clogging in a cellular automaton model for pedestrian dynamics, Phys. Rev. E 670561227 (2003).

3. Yano, R. Effect of form of obstacle on speed of crowd evacuation, Phys. Rev. E 97, 032319 (2018).

4. Huang, H.J., and Guo, R.Y., Static floor field and exit choice for pedestrian evacuation in rooms with internal obstacles and multiple exits, Phys. Rev. E 78, 021131 (2008).

5. Varas, A., Cornejo, M.D., Mainemer, D., Toledo, B., Rogan, J., Muñoz, V., and Valdivia, J.A., Cellular automaton model for evacuation process with obstacles, Physica A 382, 631-42 (2007).

6. Alizadeh, R., A dynamic cellular automaton model for evacuation process with obstacle,s Saf. Sci. 49, 315-23 (2011).

7. Frank, G.A. and Dorso, C.O., Room evacuation in the presence of an obstacle, Physica A 390, $2135-45$ (2011).

8. Helbing, D., Farkas, I.J., Molnar, P., and Vicsek, T. Simulation of pedestrian crowds in normal and evacuation situations Pedestrian and Evacuation Dynamics, Springer, Berlin, p.21 (2002).

9. Helbing, D., Buzna, L., Johansson, A., and Werner, T., Self-organized pedestrian crowd dynamics experiments, simulations, and design solutions. Transp. Sci. 39 1-24 (2005).

10. Yanagisawa, D., Kimura, A., Tomoeda, A., Nishi, R., Suma, Y., Ohtsuka, K., and Nishinari, K., Introduction of frictional and turning function for pedestrian outflow with an obstacle, Phys. Rev. E 80, 036110 (2009).

11. Jiang, L., Li, J., Shen, C., Yang, S. and Han, Z., Obstacle optimization for panic flow-reducing the tangential momentum increases the escape speed, PLoS One 9 9(12), e115463 (2014).

12. Garcimartín, Á., Maza, D., Pastor, J. M., Parisi, D. R., Martín-Gómez, C., and Zuriguel, I., Redefining the role of obstacles in pedestrian evacuation. New Journal of Physics 20(12), 123025 (2018).

13. Zhao, Y., Lu, T., Fu, L., Wu, P., and Li, M. Experimental verification of escape efficiency enhancement by the presence of obstacles. Safety science, 122, 104517 (2020).

14. Feliciani, C., Zuriguel, I., Garcimartín, A., Maza, D., and Nishinari, K. Systematic experimental investigation of the obstacle effect during non-competitive and extremely competitive evacuations. Scientific Reports 10(1), 1-20 (2020). 
15. Shiwakoti, N., Shi, X., and Ye, Z., A review on the performance of an obstacle near an exit on pedestrian crowd evacuation. Safety science 113, 54-67 (2019).

16. Zuriguel, I., Parisi, D.R., Hidalgo, R.C., Lozano, C., Janda, A., Gago, P.A., Peralta, J.P., Ferrer, L.M., Pugnaloni, L.A., Clément, E., Maza, D., Pagonabarraga, I., and Garcimartín, A., Scientific Reports 4, 7324 (2014).

17. Zuriguel, I., Olivares, J., Pastor, J.M., Martín-Gómez, C., Ferrer, L.M., Ramos, J.J., and Garcimartín, A., Effect of obstacle position in the flow of sheep through a narrow door, Phys. Rev. E 94, 032302 (2016).

18. Lin, P., Ma, J., Liu, T.Y., Ran, T., Si, Y.L., Wu, F.Y. and Wang, G.Y., An experimental study of the impact of an obstacle on the escape efficiency by using mice under high competition, Physica A 482 228-42 (2017).

19. Zuriguel, I., Janda, A., Garcimartín, A., Lozano, C., Arévalo, R., and Maza, D., Silo clogging reduction by the presence of an obstacle Phys. Rev. Lett. 107278001 (2011).

20. Lozano, C., Janda, A., Garcimartin, A., Maza, D., and Zuriguel, I., Flow and clogging in a silo with an obstacle above the orifice. Phys. Rev. E 86(3), 031306 (2012).

21. Endo, K., Anki Reddy, K., and Katsuragi, H., Obstacle-shape effect in a two-dimensional granular silo flow field. Phys. Rev. Fluids 2, 094302 (2017).

22. Reddy, A.V.K., Kumar, S., Reddy, K.A., and Talbot, J. (2018). Granular silo flow of inelastic dumbbells: Clogging and its reduction. Phys. Rev. E 98(2), 022904.

23. To, K., Lai, P.Y., and Pak, H.K., Jamming of granular flow in a two-dimensional hopper, Phys. Rev. Lett. 86, 71 (2001).

24. Zuriguel, I., Garcimartín, A., Maza, D., Pugnaloni, L.A., and Pastor, J.M., Jamming during the discharge of granular matter from a silo, Phys. Rev E 71, 051303 (2005).

25. To, K., Jamming transition in two-dimensional hoppers and silos, Phys. Rev. E 71, 060301(R) (2005).

26. Thomas, C.C. and Durian, D.J., Fraction of clogging configurations sampled by granular hopper flow, Phys. Rev. Lett. 114, 178001 (2015).

27. Gella, D., Zuriguel, I., and Maza, D. Decoupling geometrical and kinematic contributions to the silo clogging process. Phys. Rev. Lett., 121(13), 138001 (2018).

28. Gella, D., Maza, D., and Zuriguel, I. Granular flow in a silo discharged with a conveyor belt. Powder Technology 360, 104-111 (2020).

29. Janda, A., Zuriguel, I., and Maza, D., Flow rate of particles through apertures obtained from self-similar density and velocity profiles, Phys. Rev. Lett. 108, 248001 (2012).

30. Gella, D., Zuriguel, I., and Ortín, J., Multifractal intermittency in granular flow through bottlenecks. Phys Rev. Lett. 123(21), 218004 (2019).

31. Reichhardt, C.J.O. and Reichhardt, C., Avalanche dynamics for active matter in heterogeneous media. New J. Phys. 20, $025002(2018)$.

32. Leyva, S.G., Stoop, R.L., Tierno, P., and Pagonabarraga, I. Dynamics and clogging of colloidal monolayers magnetically driven through a heterogeneous landscape, ${ }^{\circ}$ textitSoft Matter, 16(30), 6985-6992 (2020).

33. Gella, D., PhD Thesis, Flow and clogging in a granular silo discharged with a conveyor belt. Universidad de Navarra, Pamplona, pp. 122-127 (2020).

34. Goldhirsch, I., Stress, stress asymmetry and couple stress: from discrete particles to continuous fields, Granular Matter 12, 239-252 (2010). 


\section{Supplementary Files}

This is a list of supplementary files associated with this preprint. Click to download.

- Protocol.mp4 